\title{
EXTRAÇÃO E PRÉ-CONCENTRAÇÃO DE COMPOSTOS ORGÂNICOS VOLÁTEIS POR PERMEAÇÃO EM MEMBRANA PẢRA ANÁLISE CROMATOGRÁFICA
}

\section{Eduardo Carasek da Rocha}

Departamento de Química - Universidade Federal de Santa Catarina (UFSC) - 88040-900 - Florianópolis - SC

Antonio Luiz Pires Valente* e Fabio Augusto

Instituto de Química - Universidade Estadual de Campinas (Unicamp) - 13083-970 - Campinas - SP

Recebido em 4/11/98; aceito em 27/4/99

\begin{abstract}
EXTRACTION AND PRE-CONCENTRATION OF VOLATILE ORGANIC COMPOUNDS BY MEMBRANE PERMEATION FOR CHROMATOGRAPHY ANALYSIS. This work discusses sample preparation processes for gas chromatography (GC) based on the technique of extraction through membrane permeation (MPE). The MPE technique may be easily coupled to GC via a relatively simple device, which is a module that holds the membrane and is directly connected to the GC column. The possibility of operational errors due to sample handling is substantially reduced in an MPE-GC system because the sample preparation and the chemical analysis are accomplished as a one-step process. The MPE technique is of relatively wide application as it can be used for aqueous samples, solid samples and gaseous samples. Depending on the type of sample the extraction is performed with the membrane in direct contact with the sample or in contact with its headspace. The MPE-GC technique is very useful in trace analysis, due to the timedependent enrichment of the analyte. A typical application of MPE-GC is the analysis of VOCs present in water that may be accomplished with detection limits at the low ppb $\left(\mu \mathrm{gL}^{-1}\right) \operatorname{level}$
\end{abstract}

Keywords: membrane permeation; volatile organic compounds; gas chromatography.

\section{INTRODUÇÃO}

A extração de analitos por permeação em membranas (EPM) como técnica de preparação de amostras para análise química foi primeiramente relatada na década de sessenta, para análises de ar e água por espectroscopia de massas (EM), utilizando-se membranas semipermeáveis planas ${ }^{1}$. Este relato motivou a continuidade de pesquisas com EPM, pela sua potencialidade como método de preparação de amostras seletivo, eficiente, livre de solventes, simples, rápido, de baixo custo e independente do tipo de instrumento utilizado. Ademais, com dispositivos adequados, a EPM permite, em uma única etapa, separar os componentes da matriz, concentrá-los e dirigí-los para o instrumento analítico, possibilitando determinações analíticas rápidas e monitoramento contínuo direto (on-line $)^{2}$. A partir de $1974^{3}$ as características de membranas tubulares vêm sendo exploradas, pois elas apresentam vantagens sobre as membranas planas para certas aplicações analíticas ${ }^{4-6}$. Apesar de três décadas de desenvolvimento de tecnologia de EPM existe pouca informação de seu uso em preparação de amostras para cromatografia gasosa $(\mathrm{CG})^{7}$. Recentemente foi demonstrado que a combinação de EPM-CG é uma promissora tecnologia para a extração e análise de compostos orgânicos voláteis (COV) de água e $\operatorname{ar}^{8}$.

No Brasil, diversos pesquisadores tem contribuído com estudos fundamentais e propostas de uso de separação por permeação em membranas para diversas aplicações. Pereira, Habert, Nóbrega e Borges ${ }^{9}$ estudaram a separação de contaminantes voláteis em águas por pervaporação através de membranas poliméricas. Mendes, Pimpim, Kotiaho, Barone e Eberlin desenvolveram um módulo de permeação em membrana para introdução seletiva de analitos em espectrômetro de massas ${ }^{10}$; uma modificação deste dispositivo foi empregada na determinação quantitativa de contaminantes em águas a nível de pg. $\mathrm{L}^{-111}$.

\section{DISPOSITIVO BÁSICO DE EPM}

Na Figura 1 está esquematizado um arranjo para preparação de amostras baseado em permeação por membrana; o arranjo consiste de um módulo de extração e um módulo de trapeamento, acoplados em série e conectados a um cromatógrafo gasoso. Como mostrado nesta figura, o módulo de extração tem uma câmara (A) no interior da qual é alojada a membrana tubular (B) de forma que o único contato desta com o material que flui na câmara ocorra pela parede externa da membrana; portanto, a membrana pode ser considerada uma segunda câmara (C). A amostra aquosa ou gasosa, flui pela câmara $\mathbf{A}$ enquanto um fluxo de gás inerte (e.g., $\mathrm{N}_{2}$ ) passa pela câmara $\mathbf{C}$. O contato da amostra com a membrana permite que compostos orgânicos - os analitos - permeiem seletivamente por sua parede, sendo dissolvidos e transportados

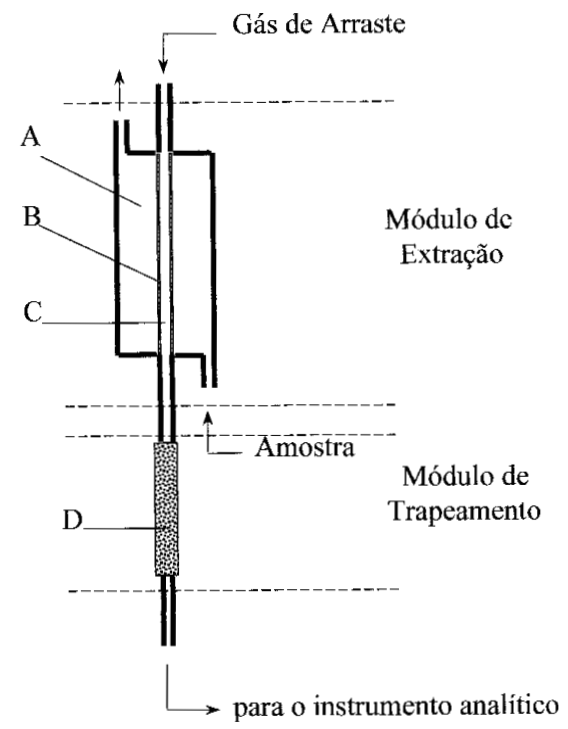

Figura 1. Esquema de um dispositivo básico para extração por permeação em membrana (EPM). 
pelo gás inerte até o módulo de trapeamento (D). Nesse módulo os analitos são retidos e concentrados, por adsorção e/ou congelamento (por exemplo, por resfriamento do módulo com nitrogênio líquido ou gêlo seco). Após um intervalo de tempo pré-determinado, cessa-se a passagem da amostra pela câmara A e aquece-se rapidamente o módulo de trapeamento, causando a dessorção do material retido e o seu transporte pelo fluxo de gás inerte para a coluna cromatográfica.

\section{Membranas e Processo de Permeação}

Duas categorias de membranas são utilizadas para o processo de permeação: membranas não porosas, como as de silicone, e membranas microporosas, como as de polipropileno ${ }^{12}$. No caso de uma membrana microporosa a permeação ocorre por difusão direta dos analitos através dos poros da membrana ${ }^{8}$. Para membranas não porosas o mecanismo de transporte dos analitos da amostra para a membrana e desta para a corrente de gás inerte ocorre em três etapas: migração dos analitos da fase aquosa para a superfície da membrana, onde eles são dissolvidos; migração pela parede da membrana, sob gradiente de concentração; e dessorção na outra face da membrana, com dissolução no gás de transporte $^{8,13}$. Pode-se demonstrar que, para membranas tubulares finas e amostras gasosas, a velocidade de transferência de massa, n, de uma determinada espécie é dada por ${ }^{7}$ :

$$
v=2 \pi \cdot \mathrm{D}_{\mathrm{f}} \cdot \mathrm{K}_{\mathrm{am}} \cdot \frac{\mathrm{R} \cdot \mathrm{L}}{\mathrm{b}} \cdot \mathrm{C}
$$

onde: $\boldsymbol{D}_{f}$ é o coeficiente de difusão $\left(\mathrm{cm}^{\left.-\mathrm{s}^{-1}\right)}\right.$ do analito no material da membrana; $\boldsymbol{K}_{a m}$ é a constante de distribuição deste analito entre a amostra e a superfície da membrana; $\boldsymbol{R}, \boldsymbol{L}, \boldsymbol{b}$ são, respectivamente, o raio, o comprimento e a espessura de parede da membrana, e $\boldsymbol{C}$ é a concentração de analito na amostra. Nesta equação pode-se observar que a velocidade de permeação de um analito depende de sua concentração na amostra, das dimensões da membrana (essencialmente, seu comprimento $L$, seu diâmetro externo $\mathbf{d}_{\mathbf{e}}$ e a espessura de suas paredes $\mathbf{d}_{\mathbf{m}}$ ) e de características físico-químicas do material da membrana e do analito.

Para aplicação da metodologia de EPM-CG com amostras que contém água é comum o uso de membranas de silicone, um material altamente hidrofóbico e onde a difusibilidade da água é muito baixa ${ }^{12}$. A hidrofobicidade das membranas de silicone, que é o fator determinante para separar seletivamente analitos orgânicos de matrizes aquosas, também é importante para a análise por CG porquê via de regra as colunas cromatográficas adequadas para a separação de compostos orgânicos podem ser degradadas por umidade. Mesmo assim, essas membranas de silicone permitem a permeação de pequenas quantidades de água, razão pela qual existe proposta de uso de membranas de novos materiais ainda mais hidrofóbicos, como os fluorosilicones ${ }^{14}$.

\section{Módulo de Extração}

Como ilustrado na Figura 2 o módulo de extração pode apresentar diferentes configurações, dependendo do tipo de amostra para a qual a EPM será aplicada. Nas Figuras 2A e 2B estão representadas extrações com a membrana tubular mergulhada na amostra, viáveis na ausência de particulados e outros materiais que possam impregnar na membrana. Na Figura $2 \mathrm{C}$ está representada uma configuração para extração do headspace, adequada para matrizes sólidas ou matrizes líquidas contendo material em suspensão. Nas configurações das Figuras 2A, 2B e 2C, o módulo de extração é bastante simples, consistindo de um pedaço de membrana tubular conectada a tubos de aço inoxidável fixados na tampa do frasco de amostragem. O gás inerte flui pelo conjunto tubo-membrana-tubo e arrasta os analitos que permeiam a membrana, oriundos de uma quantidade fixa de amostra. A configuração da Figura 2D é usada para extração dinâmica, na qual a amostra flui constantemente pelos tubos de entrada e saída de amostra. Nesse modo de extração a amostra passa pelo módulo sob vazão constante, de forma que a quantidade de amostra em contato com a membrana é determinada pelo tempo de extração ${ }^{15}$.

As Figuras 2E e 2F mostram módulos de extração de construção mais elaborada, nos quais um invólucro de vidro selado contém, além do conjunto tubo-membrana-tubo, dois outros tubos, de entrada e saída. Na configuração da Figura 2E o módulo de extração é operado no modo denominado de flow over, no qual o gás de transporte flui internamente ao conjunto tubomembrana-tubo, enquanto a amostra aquosa flui exteriormente em contracorrente - ou "sobre" a membrana. A configuração da Figura 2F é para operação no modo flow through, em que o gás de transporte flui externamente à membrana e a amostra internamente, também em contracorrente.
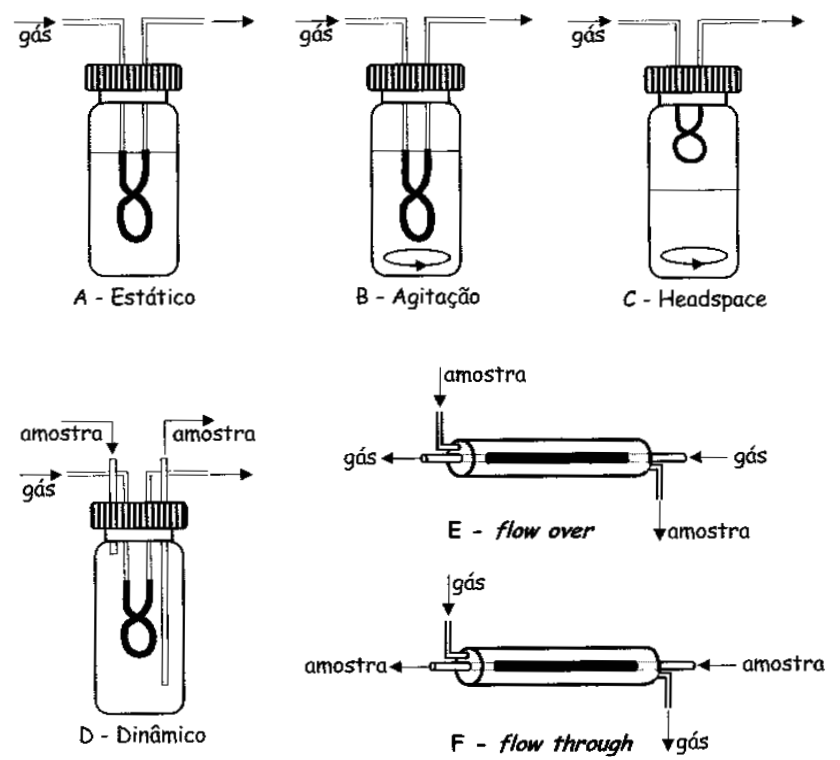

Figura 2. Configurações do módulo de extração de EPM utilizadas experimentalmente (detalhes no texto)

Para extrações quantitativas de COV de águas alguns parâmetros operacionais do módulo de extração devem ser otimizados. Particularmente, no caso das configurações flow through e flow over deve-se controlar o tempo de contato entre a amostra e a membrana, a vazão e a temperatura da amostra, efeitos de força iônica (salificação) e do $\mathrm{pH}$ da amostra, além de um intervalo de tempo adicional após encerrada a passagem da amostra pelo módulo de extração, que tem por objetivo permitir a permeação dos analitos que continuam na parede da membrana ao cessar a passagem da amostra. A influência destes parâmetros sobre a eficiência de extração de COV foi reportada para módulos de extração de diversas dimensões ${ }^{12,14}, 16$.

\section{Módulo de Trapeamento}

No módulo de trapeamento os analitos permeados pela membrana são concentrados e, em seqüência, dessorvidos para rápida introdução no sistema cromatográfico. Esse módulo deve incorporar dispositivos de aquecimento rápido, para a dessorção dos materiais extraídos, e eventualmente pode ter recursos de resfriamento a temperaturas sub-ambiente, para permitir trapeamento crioscópico. 
O módulo de trapeamento pode ser simplesmente uma seção do início da própria coluna cromatográfica ${ }^{15}$ - o material sorvente é a fase estacionária da coluna - ou tubos conectados diretamente à ponta da coluna cromatográfica, recheados com sorventes como Tenax ou carvão ativo ${ }^{17}$. Nos dois casos a eficiência de trapeamento pode ser aumentada pelo resfriamento do módulo. Quando o trapeamento é feito em seção da coluna cromatográfica ou em tubos com materiais sorventes, o método de EPM é conhecido como Extração por Membrana com Interface Sorvente, MESI (de Membrane Extraction with Sorbent Interface $)^{2}$. Uma alternativa ao sistema MESI, na qual o módulo de trapeamento é constituido simplesmente por um tubo capilar de material inerte e isento de sorvente, onde os analitos são retidos apenas por efeito de resfriamento, é denominada Permeação em Membrana com Trapeamento Crioscópico, PMTC. Já foi reportado o uso de PMTC usando como módulo de trapeamento capilares de vidro ${ }^{14}$ ou tubos finos de Silcosteel ${ }^{16}$ (aço inoxidável recoberto com uma monocamada de sílica).

Os módulos de trapeamento para MESI e PMTC devem ser projetados para ter baixa resistência térmica, de forma a permitir aquecimento e liberação rápida dos analitos para a coluna cromatográfica. No sistema PMTC é recomendável utilizar tubos de materiais de baixa ou nenhuma interação com os analitos, para evitar os efeitos de memória que geralmente ocorrem com os materiais sorbentes ${ }^{12}$.

\section{O USO DE EPM NA PREPARAÇÃO DE AMOSTRAS}

A partir de 1990 têm sido relatados trabalhos com EPMCG para a determinação de compostos orgânicos voláteis e semivoláteis em águas, solos e ar, utilizando os sistemas MESI e PMTC, principalmente para as configurações flow over e flow through. Nestes trabalhos são descritas boas reprodutibilidades e limites de detecção para COV abaixo de $\mu \mathrm{g} . \mathrm{L}^{-1}$, com tempos de extração variando de 2 a 40 minutos. Compostos voláteis de óleos essenciais também podem ser analisados por EPM-CG ${ }^{18}$.

Mitra e Guo ${ }^{19}$, propuseram EPM-CG-DIC (Detecção por Ionização em Chama) na configuração flow through como alternativa a métodos padrão da EPA (Environmental Protection Agency dos EUA) para a quantificação de hidrocarbonetos aromáticos e halogenados em águas. O módulo de extração do sistema estudado tinha uma membrana tubular de silicone $\left(\mathrm{L}=20 \mathrm{~cm} \mathrm{x} \mathrm{d} \mathrm{e}_{\mathrm{e}}=635 \mu \mathrm{m} \times \mathrm{d}_{\mathrm{m}}=165\right.$ $\mu \mathrm{m})$, e o módulo de trapeamento um tubo de aço de $15 \mathrm{~cm}$ de comprimento e $0,52 \mathrm{~mm}$ de diâmetro interno recheado com Carbotrap $C$ (carvão ativo grafitizado). Nitrogênio foi usado como gás de transporte. Os limites de detecção obtidos foram significativamente melhores que os dos métodos padrão correspondentes; p.ex., 1,2 ng. $\mathrm{L}^{-1}$ para benzeno, contra $40 \mathrm{ng} . \mathrm{L}^{-1}$ no método EPA 8260 e 110 ng.L $\mathrm{L}^{-1}$ no método EPA 524.2.

Um módulo de extração, operado na configuração flow through e constituído por uma membrana de silicone extremamente longa $\left(\mathrm{L}=110 \mathrm{~cm} \mathrm{x} \mathrm{d} \mathrm{d}_{\mathrm{e}}=650 \mu \mathrm{m} \times \mathrm{d}_{\mathrm{m}}=350 \mu \mathrm{m}\right)$ conectada a tubos capilares de sílica fundida de $450 \mu \mathrm{m}$ de diâmetro externo foi usado para a análise por CG-DIC de sete COV em águas com concentrações de $1000 \mu \mathrm{g} . \mathrm{L}^{-1}$ e 10 $\mu \mathrm{g} . \mathrm{L}^{-114}$. O módulo de trapeamento crioscópico era um tubo de vidro de $60 \mathrm{~mm}$ de comprimento e $1,0 \mathrm{~mm}$ de diâmetro interno resfriado por nitrogênio líquido. $O$ tempo de extração foi $11 \mathrm{~min}$, sendo que hélio foi utilizado como gás de transporte. Como mostrado na Tabela 1, na maioria dos casos as quantificações tiveram incertezas apropriadas para análise quantitativa, com estimativas de desvio padrão relativo entre $0,6 \%$ e $3,7 \%$. A Figura 3 mostra um dos cromatogramas obtidos para amostra contendo $10 \mu \mathrm{g} . \mathrm{L}^{-1}$ de cada COV analisado.
Tabela 1. Repetibilidades, expressas como estimativas de desvio padrão relativos $s_{R}$ em \%, obtidas em análises EPM-CG de sete COV em água em dois níveis de concentração ${ }^{14}$.

\begin{tabular}{|c|c|c|}
\hline \multirow[b]{2}{*}{ Composto } & \multicolumn{2}{|c|}{$S_{R}(\%)^{a}$} \\
\hline & $C=1000 \mu$ g. $L^{-1}$ & $\mathrm{C}=10 \mu \mathrm{g} . \mathrm{L}^{-1}$ \\
\hline Diclorometano & 1,45 & 3,72 \\
\hline Clorofórmio & 0,61 & 1,10 \\
\hline Benzeno & 0,83 & 1,31 \\
\hline Heptano & 2,38 & 8,52 \\
\hline Tolueno & 1,46 & 1,12 \\
\hline Tetracloroetileno & 0,95 & 1,77 \\
\hline p-Xileno & 0,98 & 0,61 \\
\hline
\end{tabular}

Nota: (a) Número de replicatas $=5$.

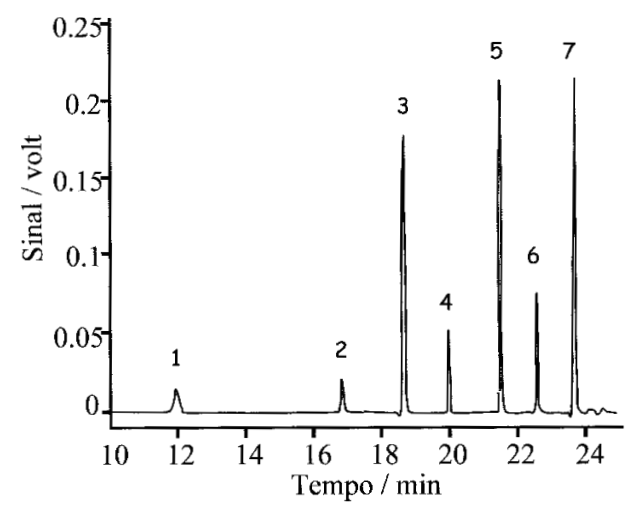

Figura 3. Resultado típico de Análise por PMTC-CG de sete compostos orgânicos voláteis (COV) em água, todos na concentração de 10 $m g . L^{-1}$. (1) diclorometano, (2) clorofórmio, (3) benzeno, (4) heptano, (5) tolueno, (6) tetracloroetileno e (7) p-xileno.

Pawliszyn e Yang ${ }^{20}$ construiram módulo de extração, para operar nas configurações flow over e flow through, utilizando tanto membranas de silicone $\left(\mathrm{L}=8 \mathrm{~cm} \mathrm{x} \mathrm{d} \mathrm{e}_{\mathrm{e}}=305 \mu \mathrm{m} \mathrm{x} \mathrm{d}\right.$ $165 \mu \mathrm{m})$ como de polipropileno $\left(\mathrm{L}=8 \mathrm{~cm} \mathrm{x} \mathrm{d}=100 \mu \mathrm{m} \times \mathrm{d}_{\mathrm{m}}\right.$ $=30 \mu \mathrm{m})$, montadas no interior de um tubo de vidro de $10 \mathrm{~cm}$ de comprimento e $1,8 \mathrm{~mm}$ de diâmetro interno. O módulo de trapeamento era um pedaço de $8 \mathrm{~cm}$ da própria coluna cromatográfica, resfriada com gêlo seco durante a extração. Nos testes com membrana de polipropileno, um tubo recheado com material secante (Nafion) foi inserido entre os módulos de extração e trapeamento, para retenção da água permeada. O gás de transporte usado foi hélio. Para tricloroetileno, obtiveram-se limites de detecção de $1 \mu \mathrm{gL}^{-1}$ e estimativas de desvio padrão relativo inferiores a $3 \%$.

Além de águas, EPM-CG também pode ser aplicada a outras matrizes. Bryce, Izquierdo e Castro ${ }^{21}$ descreveram um sistema EPM-CG, com Detecção por Fluorescência Atômica, para determinação de compostos organomercurícos em solos e assimilados. O módulo de extração usava uma membrana plana de silicone, e o módulo de trapeamento consistia em uma microcoluna recheada com Tenax. Os limites de detecção reportados foram de $0,25 \mu \mathrm{g} . \mathrm{Kg}^{-1}$ para $\mathrm{Me}_{2} \mathrm{Hg}, 0,41 \mu \mathrm{g} \cdot \mathrm{Kg}^{-1}$ para $\mathrm{Et}_{2} \mathrm{Hg}$ e $255 \mu \mathrm{g} . \mathrm{Kg}^{-1}$ para $\mathrm{MeHgCl}$, com estimativas de desvio padrão relativo entre $1,3 \%$ e $7,2 \%$. Um sistema similar, mas com Detecção por Captura de Eletrons, foi aplicado em análise de hidrocarbonetos halogenados em solo ${ }^{22}$, sendo detectáveis 1,0 $\mu \mathrm{g} . \mathrm{Kg}^{-1}$ de clorofórmio, $0,6 \mu \mathrm{g} \cdot \mathrm{Kg}^{-1}$ de tetracloreto de carbono e $4,4 \mu \mathrm{g} \cdot \mathrm{Kg}^{-1}$ de tricloroeteno.

Uma modificação interessante de EPM é o chamado modo de dessorção pulsada ${ }^{17,23}$. No procedimento convencional de EPM, os analitos extraídos de uma amostra são totalmente 
dessorvidos e cromatografados por aquecimento prolongado do módulo de dessorção. No modo pulsado, o material extraído de uma alíquota de amostra é apenas parcialmente dessorvido por um pulso rápido de aquecimento do módulo de extração, sendo a fração dessorvida cromatografada. Para cada alíquota de amostra pode ser feita uma extração e várias dessas dessorções parciais, cada uma delas gerando um cromatograma usado como replicata da análise. A Tabela 2 lista limites de detecção obtidos para diversos COV quantificados em água com EPM-CGDIC no modo de dessorção pulsada ${ }^{17}$.

Tabela 2. Limites de detecção $\mathrm{LD}$, em $\mathrm{mg} \cdot \mathrm{L}^{-1}$ a , obtidos por EPM-CG para seis COV extraídos de água ${ }^{17}$, no modo de dessorção pulsada.

\begin{tabular}{cc}
\hline Composto & LD \\
\hline Tolueno & 0,042 \\
Tricloroetano & 0,28 \\
Hexano & 1,45 \\
Diclorometano & 7,75 \\
Acetona & 61,1 \\
Etanol & 212 \\
\hline
\end{tabular}

Nota: (a) Relação sinal/ruído igual a 3.

É possível a ocorrência de efeitos de memória causado por resíduos de analitos dissolvidos na membrana após a análise, especialmente em amostras contendo analitos pouco voláteis e em concentrações altas (da ordem de centenas de $\mu \mathrm{g} . \mathrm{L}^{-1}$ ). Assim, entre cada extração a membrana deve ser limpa; isso pode ser feito pela passagem de água no módulo de extração por até $80 \min ^{15,20,24}$. Como alternativa à água como fluido de limpeza, Rocha, Augusto e Valente propuseram nitrogênio gasoso ${ }^{25}$, tendo sido feitos testes com este gás à temperatura ambiente e com aquecimento a $80^{\circ} \mathrm{C}$. No sistema MESI empregado nos testes, operável nos modos flow over ou flow through, o módulo de trapeamento era uma membrana de silicone $\left(\mathrm{L}=1,5 \mathrm{~cm} \mathrm{x} \mathrm{d}=196 \mu \mathrm{m} \mathrm{x} \mathrm{d} \mathrm{d}_{\mathrm{m}}=24 \mu \mathrm{m}\right)$ montada no interior de um tubo de vidro de $3 \mathrm{~cm}$ de comprimento e 6 $\mathrm{mm}$ de diâmetro externo. O módulo de trapeamento crioscópico era constituido por um tubo de Silcosteel com 7 $\mathrm{cm}$ de comprimento e 1,6 $\mathrm{mm}$ de diâmetro externo. Conseguiu-se limpeza completa da membrana em menos de $20 \mathrm{~min}$, inclusive para compostos menos voláteis que o 1,2dicloroetano e em concentrações acima de $20 \mathrm{mg} . \mathrm{L}^{-1}$; a Figura 4 permite comparar a eficiência da limpeza da membrana por essa técnica em relação às metodologias convencionais. Este sistema foi aplicado para análises de COV em águas com concentrações variando de pg. $\mathrm{L}^{-1}$ a mg. $\mathrm{L}^{-1}$, com estimativas de desvio padrão relativo inferiores a $5 \%$.

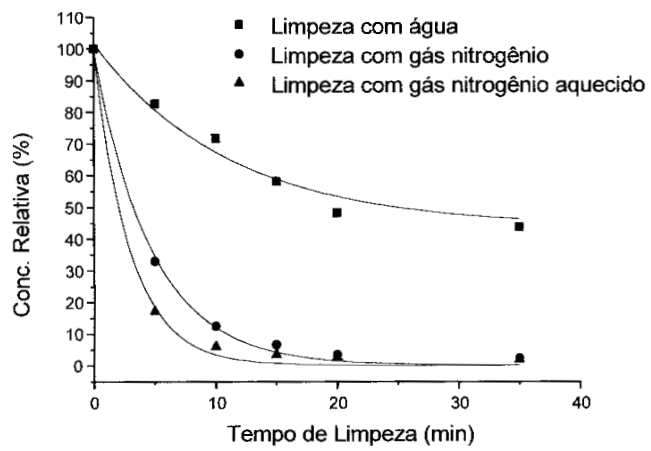

Figura 4. Eficiências de limpeza de membrana de silicone com três procedimentos: passagem de água pura, de nitrogênio a temperatura ambiente e de nitrogênio na temperatura de $80^{\circ} \mathrm{C}$.

\section{CONCLUSÕES}

A extração por permeação em membrana, EPM, é uma técnica de preparo de amostras simples e de boa repetibilidade, aplicável para análises rápidas de rotina ou monitoramento contínuo de compostos orgânicos em matrizes ambientais ou industriais. A construção dos módulos de EPM é simples, de baixo custo e pode ser realizada no laboratório. Minimizar os tamanhos dos módulos facilita o seu acoplamento ao cromatógrafo gasoso sem modificação do injetor do aparelho. A técnica de EPM-CG pode ser automatizada, com o que adquire grande potencial para a aplicação em análises de monitoramento contínuo. A EPM é totalmente livre do uso de solventes, que podem ter pureza inadequada para trabalhos de análises de traços e gerar problemas de uso e descarte. Como na EPM-CG a amostra é preparada em uma única etapa evitam-se contaminações devidas à sua manipulação. Deste modo, a EPM-CG apresenta características que a tornam um método de grande potencial para a preparação de amostras para análise por cromatografia gasosa.

\section{AGRADECIMENTOS}

À FAPESP e CNPq pelos suportes que permitiram a organização deste artigo.

\section{REFERÊNCIAS}

1. Hoch, G.; Kok, B.; Arch. Biochem. Biophys. 1963, 101, 160

2. Yang, M. J.; Harms, S.; Luo, Y. Z.; Pawliszyn; J.; Anal. Chem. 1994, 66, 339

3. Westover, L. B.; Tou, J. C.; Mark, J. H.; Anal. Chem. 1974, 46, 568

4. Slivon, L. E.; Baner, M. R.; Ho, J. S.; Budde, W. L.; Anal. Chem. 1991, 63, 1335

5. Lapack, M. A.; Tou, J. C.; Enke, C. G.; Anal. Chem. 1990, 62,1265

6. Brodbelt, J. S.; Cooks, R. G.; Tou, J. C.; Kallos, G. J.; Dryzga, M. D.; Anal. Chem. 1987, 59, 454

7. Yang, M. J.; Luo, Y. Z.; Pawliszyn, J.; Chemtech 1994, 10,31

8. Kurán, P.; Soják, L.; J. Chromatogr. A 1996, 733, 119

9. Pereira C. C.; Habert A. C.; Nobrega R.; Borges C. P.; J. Membr. Sci. 1998, 138, 227.

10. Mendes M. A.; Pimpim R. S.; Kotiaho T.; Barone J. S.; Eberlin M. N.; Quim. Nova 1996, 19, 480.

11. Mendes M. A.; Pimpim R. S.; Kotiaho T.; Eberlin M. N.; Anal. Chem. 1996, 68, 3502.

12. Mitra, S.; Xu, Y. H.; J. Chromatogr. A 1994, 688, 171

13. Mitra, S.; Zhu, N.; Zhang, X.; Kebbekus, B.; J. Chromatogr. A 1996, 736, 165.

14. Burger, B. V.; Burger, W. J .G.; Burger, I.; J. High Resolut. Chromatogr. 1996, 19, 571

15. Yang, M. J.; Pawliszyn, J.; Anal. Chem. 1993, 65, 1758

16. Rocha, E. C.; Tese de Doutorado; Unicamp, Campinas, 1997

17. Mitra, S.; Chen, Y.; J. Chromatogr. A 1993, 648, 415

18. Rocha, E. C.; Pini, G. F.; Valente, A. L. P.; Livro de Resumos - IX Encontro Nacional de Química Analítica, São Carlos (SP), p. 209, 1997.

19. Mitra, M.; Guo, X.; Anal. Lett. 1998, 31, 367.

20. Yang, M. J.; Harms, S.; Luo, Y. Z.; Pawliszyn, J.; Anal. Chem. 1994, 66, 1339

21. Bryce, D. W.; Izquierdo, A.; Castro, M. D. L.; Anal. Chem. 1997, 69, 844.

22. Papaefstathiou, M. T.; Tena, M. T.; Castro, M. D. L.; J. Chromatogr. A 1997, 779, 352.

23. Mitra, M.; Guo, X.; J. Chromatogr. A 1998, 826, 39.

24. Koester, C. J.; Clement, R. E.; Crit. Rev. Anal. Chem. 1993, 24, 262

25. Rocha, E. C.; Augusto, F.; Valente, A. L. P.; J. Microcolumn Sep. 1999, 11, 29. 